\title{
Preparation of Dipalmitoyl Phosphatidycholine-Modified Chitosan Nanostructures and Its Application for Enhancing Chromium Adsorption
}

\author{
Ruochen Wang, ${ }^{\dagger, a, b}$ Fan Yi, ${ }^{\dagger, a, b}$ Yu Qiao, ${ }^{a, b}$ Gang Huang, ${ }^{*, c}$ Jun Zhu, ${ }^{*, b}$ and Dannong He $\mathrm{e}^{*, a, b}$ \\ a School of Materials Science and Engineering, Shanghai Jiao Tong University, 800 Dongchuan Road, Shanghai 200240, China \\ ${ }^{b}$ National Engineering Research Center for Nanotechnology, 28 East Jiangchuan Road, Shanghai 200241, China \\ ${ }^{c}$ Shanghai University of Medicine \& Health Sciences, 279 Zhouzhu Road, Shanghai 201318, China
}

Email: hdn_nercn@163.com (D. H.), yzjzhu@163.com (J. Z.), huang2802@163.com (G. H.)

${ }^{\dagger}$ These authors contributed equally to this work.

\begin{abstract}
Chitosan (CTS) nanospheres are successfully modified dipalmitoyl phosphatidycholine (DPPC) through by solvent removal. The physicochemical properties are characterized by dynamic light scattering (DLS), transmission electron microscope (TEM) and fourier transform infrared spectroscopy (FT-IR). Furthermore, the CTS-DPPC nanospheres are used as a novel biosorbent for the application in $\mathrm{Cr}(\mathrm{VI})$ absorption, and the effect of $\mathrm{pH}$ and additional anions on the $\mathrm{Cr}(\mathrm{VI})$ adsorption performance is evaluated. Additionally, CTS-DPPC nanofibers are produced by electrospinning to improve adsorption capacity. The results show that the order of $\mathrm{Cr}(\mathrm{VI})$ adsorption performance is CTS-DPPC nanofibers, CTS-DPPC nanospheres and CTS nanospheres, which suggests DPPC is helpful for CTS to remove metal ion. Moreover, X-ray photoelectron spectrophotometer (XPS) analyses indicate that both amino and hydroxyl groups of CTS-DPPC nanospheres are engaged in the adsorption.
\end{abstract}

Keywords chitosan, dipalmitoyl phosphatidycholine, nanosphere, nanofiber, $\mathrm{Cr}(\mathrm{VI})$ adsorption

\section{Introduction}

The pulmonary surfactant (PS) is a basic component in lung, which plays an important role during many physiological processes. Additionally, PS can increase lung compliance, reduce the surface tension, anti-oxidation, anti-infection, promote discharge of intrapulmonary foreign particles and protect the lung again injury. For example, Paola ${ }^{[1]}$ reported PS had the positive effect on the bronchopulmonary dysplasia. Recently, some research on the interaction between PS and PM 2.5 is reported. For example, Kendall ${ }^{[2]}$ reported that PS can resolve the toxic PM2.5 particles by pimelosis. Specially, PS can act on heavy metal ions from PM2.5 particles, which can alleviate the lung disease, even the whole body harm by blood circulation. Concretely, there are a lot of heavy metal elements in PM2.5 particles, which can penetrate alveolar and then deposite on the surface epithelial cell after the interaction with PS. ${ }^{[3]}$ During the process, the endoplasmic reticulum will secrete more fatty acid and acetyl coenzyme A carboxylase, which would lead to the transformation from acetyl coenzyme A to malonyl coenzyme A. What's more, diglyceride acyltransferase and hormone-sensitive lipase will also promote the increase of triglycerides. ${ }^{[4,5]}$ All of immune response will promote to absorb the toxic PM2.5 particles. However, the interaction mechanism between PS and heavy metal ions is still unclear.

The treatment technologies of heavy metal ions have been well developed, including precipitation, ${ }^{[6]}$ adsorption, ${ }^{[7]}$ ion exchange ${ }^{[8]}$ reverse osmosis, ${ }^{[9]}$ electrodialysis, ${ }^{[10]}$ and so on. Among them, the biosorbent is popular. For example, chitosan (CTS) has received considerable interest and been extensively reported because of the presence of an amino group of the 2-amino-2-deoxy-glucose (glucosamine) unit. ${ }^{[11]} \mathrm{Sag}^{[12]}$ used natural CTS to remove metal ions, and Ahmad ${ }^{[13]}$ used CTS to remove the oil of waste water. Furthermore, many modified
CTS have been obtained so as to win the optimized operation conditions and absorption property. For example, Wang ${ }^{[14]}$ used CTS molecules and collagen fiber to prepare a new type of adsorbent to remove heavy metal ions of waste water. Nalini ${ }^{[15]}$ showed the crosslinking chitosan microspheres to remove the $\mathrm{Cr}(\mathrm{VI})$ in water. However, the active biology constituent has rarely been combined with CTS to improve adsorption performance.

In order to understand the interaction mechanism between PS and heavy metal ions and to develop a novel biosorbent, CTS is modified by $\mathrm{PS}$ in present experiment to absorb $\mathrm{Cr}(\mathrm{VI})$, a main ingredient of PM2.5. The effect of $\mathrm{pH}$ and additional anions on the $\mathrm{Cr}(\mathrm{VI})$ adsorption performance was evaluated, and their adsorption mechanism was proved by XPS. The research provides a potential method for shielding PM2.5 in biomedical applications.

\section{Experimental}

\section{Materials and chemicals}

Chitosan powder, sodium tripolyphosphate (TPP), potassium dichromate, ethyl alcohol, acetic acid, sodium chloride, sodium nitrate and sodium sulfate were purchased from Sinopharm Chemical Reagent Co., Ltd (Shanghai, China). Dipalmitoyl phosphatidycholine (DPPC) was purchased from Sinopharm Chemical Reagent Co., Ltd (Shanghai, China).

\section{Synthesis of CTS nanospheres}

CTS nanospheres were prepared by ionic gelation according to the previous reports as the following: ${ }^{[16]} 50 \mathrm{mg}$ of CTS powder was dissolved in $4 \mathrm{~mL}$ of $1 \%$ acetic acid solution at room temperature. Furthermore, the $\mathrm{pH}$ value of the resultant solution was adjusted to 3 using $0.1 \mathrm{~mol} \cdot \mathrm{L}^{-1} \mathrm{NaOH}$ solution after being filtered by 0.44 and $0.22 \mu \mathrm{m}$ of filter paper in order After that, $5 \mathrm{mg}$ of sodium TPP was dissolved in $5 \mathrm{~mL}$ of dou- 
ble-distilled water at room temperature and the resultant solution $(1 \mathrm{mg} / \mathrm{mL})$ was filtered as described above. Then, $1.5 \mathrm{~mL}$ of TPP solution was added to $4 \mathrm{~mL}$ of CTS solution under continuous stirring for $30 \mathrm{~min}$. The resulting products were collected by centrifuging, washing with water three times and then lyophilized.

\section{Preparation of CTS-DPPC nanospheres}

CTS-DPPC nanospheres were prepared as following steps: CTS nanospheres were dissolved in $1 \%$ acetic acid solution. Then $50 \mathrm{mg}$ of DPPC powder was dissolved in ethyl alcohol and stirred for $2 \mathrm{~h}$ at $40^{\circ} \mathrm{C}$. Next, the DPPC solution was slowly added into the CTS nanospheres solution and stirred at $40{ }^{\circ} \mathrm{C}$ with constant speed until the ethyl alcohol was totally evaporated. The resulting products were collected and lyophilized.

\section{Preparation of CTS-DPPC nanofibers}

CTS-DPPC nanofibers were prepared by electrospinning. $100 \mathrm{mg}$ of CTS and $50 \mathrm{mg}$ of DPPC powders were dissolved in acetic acid. Then the CTS-DPPC solution was drawn into a 5 $\mathrm{mL}$ syringe with a metal capillary tip of $0.7 \mathrm{~mm}$ inner diameter. Finally, the solution was ejected from the syringe using a feed rate of $0.1 \mathrm{~mm} / \mathrm{min}$, an applied voltage varying from $20 \mathrm{kV}$, and the tip-to-collector distance varying from $8 \mathrm{~cm}$. The products were collected on an aluminum foil continuously.

\section{Characterization}

Malvern Zetasizer Nano ZS (Malvern Instruments Ltd., Malvern, Worcestershire UK) based on dynamic light scattering was used to characterize the nanosphere size. The morphology of the samples was obtained by using a field-emission scanning electron microscope (FESEM, Hitachi, S-4800). Transmission electron microscope (TEM) images were recorded on a JEOL-2100F instrument using an accelerating voltage of 200 $\mathrm{kV}$. Fourier transform infrared spectroscopy (FT-IR) (Nicolet 6700 FT-IR Spectrometer) was used to characterize the chemical change of the particles before and after $\mathrm{Cr}(\mathrm{VI})$ adsorption. Surface information was detected by $\mathrm{X}$-ray photoelectron spectrophotometer (XPS), and $\mathrm{C}, \mathrm{N}, \mathrm{O}$ and $\mathrm{Cr}(\mathrm{VI})$ on each sample were scanned. Determination of the chromium concentration in a sample was performed by Varian ICP 710 Inductively coupled Plasma-Mass Spectrometry (ICP-MS) analysis.

\section{$\mathrm{Cr}(\mathrm{VI})$ adsorption}

Potassium dichromate was used to prepare $\mathrm{Cr}(\mathrm{VI})$ stock solution, and a series of solutions with $50-500 \mathrm{mg} / \mathrm{L} \mathrm{Cr}(\mathrm{VI})$ were prepared. For adsorption experiments, $0.05 \mathrm{~g}$ of adsorbent was weighed and put into $50 \mathrm{~mL} \mathrm{Cr}(\mathrm{VI})$-containing solution in a reagent bottle. The bottle was then put on a shaking plate (150 rpm) mounted in a water bath for temperature control $\left(25^{\circ} \mathrm{C}\right)$ till the adsorption reached equilibrium. After that, $10 \mathrm{~mL}$ solution was taken from the bottle and put in a centrifuge tube, then transferred to a centrifuge to separate the solid and the liquid for further analysis. The rotation rate of the centrifuge was set at $9000 \mathrm{rpm}$, and 5 min was allowed to guarantee complete separation. The supernatant was taken to determine the remaining chromium concentration. The adsorption capacity $(Q)$ of the sorbent was calculated using the following expression:

$$
Q=\frac{\left(C_{0}-C_{f}\right) V}{M}
$$

where $Q$ is the amount adsorbed $(\mathrm{mg} / \mathrm{g}), C_{0}$ and $C_{\mathrm{f}}$ are the initial and final metal ion concentrations (mg/L), respectively; $V$ is the solution volume $(\mathrm{L})$ and $M$ is the amount of adsorbent $(\mathrm{g})$ used.

\section{Results and Discussion}

DPPC is the most important content in PS and has been used to absorb many metal ions. ${ }^{[17]}$ Therefore, to evaluate the interaction between PS and PM2.5 particles, the absorption of metal ions by DPPC was performed.

As shown in Figure 1, FT-IR spectra were used to characterize the change of DPPC before and after absorbing $\mathrm{Cr}(\mathrm{VI})$. In curve a of the spectra of DPPC, the typical absorption peaks at $3100-2800 \mathrm{~cm}^{-1}$ are associated with the $\mathrm{C}-\mathrm{H}$ stretching vibration of phospholipid acyl chain, including the symmetrical and asymmetrical stretching vibrations of $\mathrm{CH}_{2}$. As shown in the characteristic peaks of the phosphatidyl polar group of DPPC in the range of $1800-700 \mathrm{~cm}^{-1}$, the bands at 1238 and $1085 \mathrm{~cm}^{-1}$ are assigned to the asymmetric and symmetric stretching vibration of $\mathrm{PO}_{2}^{-}$, the bands at 967 and $918 \mathrm{~cm}^{-1}$ are produced by the asymmetric and symmetric stretching vibration of $\mathrm{N}^{+}\left(\mathrm{CH}_{3}\right)_{3}$, and the bands at 815,768 and $715 \mathrm{~cm}^{-1}$ are characteristic of the P-O-C anti-symmetric and symmetric stretching vibrations. ${ }^{[18]}$ As can be seen from curve $b$, when $\mathrm{Cr}(\mathrm{VI})$ was introduced into the DPPC molecule, the vibration of $\mathrm{PO}_{2}^{-}$and $\mathrm{N}^{+}\left(\mathrm{CH}_{3}\right)_{3}$ moved to the low wavenumbers of 1223 and 1062 $\mathrm{cm}^{-1}$, and 1223 and $1062 \mathrm{~cm}^{-1}$, respectively. It is attributed to the presence of strong electrostatic interaction between metal ions and polar hydrophilic groups $\mathrm{PO}_{2}^{-}$, which induces average of negative charge in $\mathrm{O}=\mathrm{P}_{-} \mathrm{O}^{-}$. As a result, the IR spectra showed a decreased vibration wavenumber of $v_{\text {as }}\left(\mathrm{PO}_{2}^{-}\right) \cdot{ }^{[19]}$ Thus, these results indicate that the presence of strong interaction between $\mathrm{Cr}(\mathrm{VI})$ and polar hydrophilic groups of DPPC.

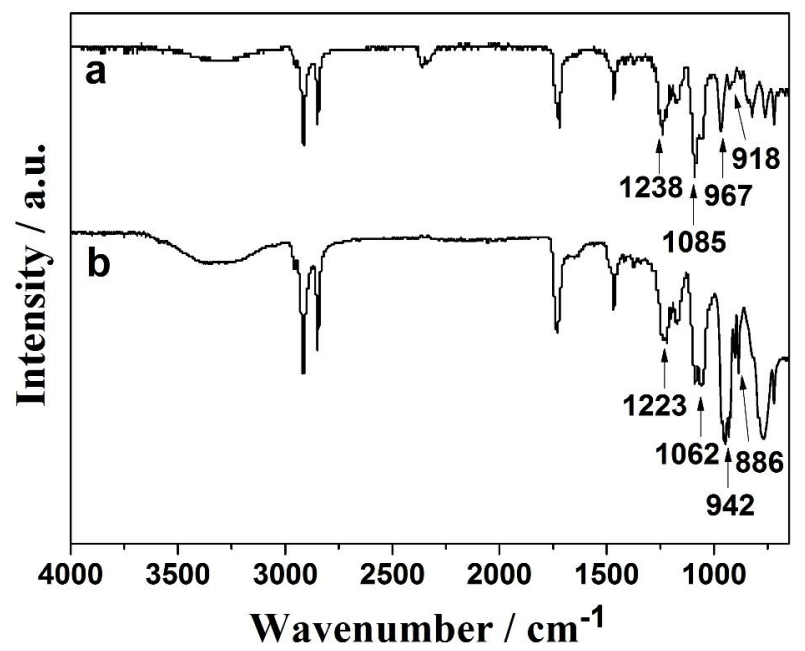

Figure 1 FT-IR spectra of DPPC before (curve a) and after (curve b) absorbing $\mathrm{Cr}(\mathrm{VI})$.

Furthermore, the complexation between CTS and DPPC was prepared to absorb $\mathrm{Cr}(\mathrm{VI})$. The DPPC modified CTS nanospheres (DPPC-CTS) was synthesized by solvent removal. As shown in Figure 2a, TEM image shows DPPC-CTS nanospheres with core in gray and shell in dark are uniform nanospheres with an average diameter of about $60 \mathrm{~nm}$, which suggests that DPPC molecules could be coated on the surface of CTS nanospheres. Moreover, Figure $2 b$ shows that the average hydrodynamic size of the CTS and DPPC-CTS nanospheres in deionized water is about 190 and $220 \mathrm{~nm}$, respectively, which reveals the DPPC addition has little influence on the size of the nanospheres. As shown in Figure 2c, the zeta potential values before and after introducing DPPC are 40.5 and $20.3 \mathrm{mV}$, respectively. Additionally, the addition of DPPC is further evidenced by FT-IR spectra (Figure 2d). In the spectra of CTS nanospheres, the typical peaks at 1635 and $1535 \mathrm{~cm}^{-1}$ are attributed to characteristic stretching vibrations of $\mathrm{C}=\mathrm{O}$ and C-N, respectively, where the band at 3200, 1070 and 1020 $\mathrm{cm}^{-1}$ is the characteristic stretching and bending vibration of 
$\mathrm{N}-\mathrm{H}$. Compared to the spectra of CTS nanospheres, the typical peaks at about 1200 and $960 \mathrm{~cm}^{-1}$ (labeled by asterisks) can be obviously found in the spectra of CTS-DPPC nanosphere, which shows the successful addition of DPPC on CTS nanospheres. The above results show that the object product is prepared successfully in our experiment.
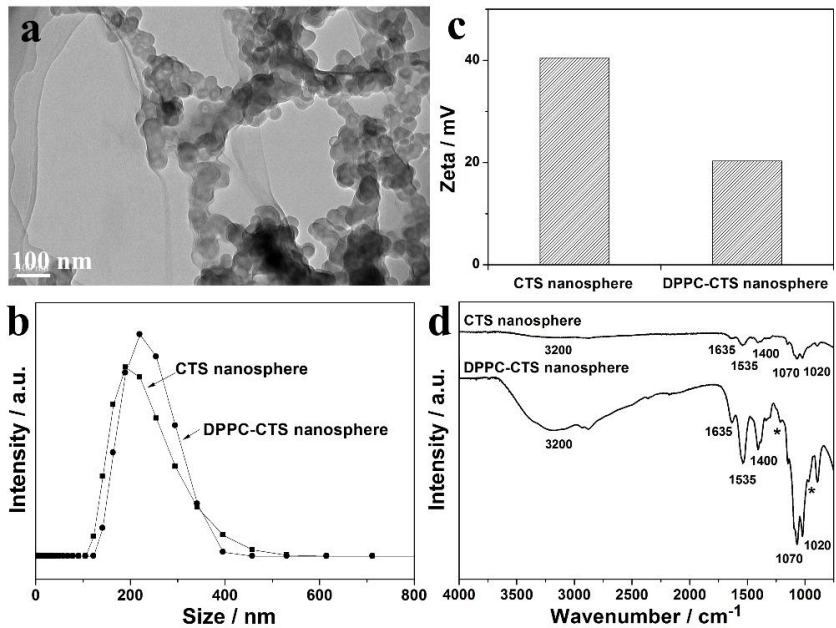

Figure 2 (a) TEM image of DPPC-CTS nanospheres, (b) the average hydrodynamic size, (c) the zeta potential value and (d) FT-IR spectra of CTS and DPPC-CTS nanospheres.

To estimate the absorption ability of CTS-DPPC nanospheres, an absorption treatment of CTS and CTS-DPPC nanospheres to $\operatorname{Cr}(\mathrm{VI})$ was detected with the increasing absorption time. As shown in Figure 3a, the adsorption capacity of CTS and CTS-DPPC nanospheres to $\mathrm{Cr}(\mathrm{VI})$ increases as a function of time at $0-2 \mathrm{~h}$. About 33.8 and $44.6 \mathrm{mg} / \mathrm{g}$ of $\mathrm{Cr}(\mathrm{VI})$ are absorbed by CTS and CTS-DPPC nanospheres after $2 \mathrm{~h}$ exposure, respectively. The result proves that DPPC is helpful for CTS to remove metal ion. Moreover, there is a slight increase of the $\mathrm{Cr}(\mathrm{VI})$ adsorption capacity for CTS-DPPC and CTS nanospheres, which seems to reach adsorption equilibrium for both CTS and CTS-DPPC nanospheres. About 34.5 and $46.2 \mathrm{mg} / \mathrm{g}$ of $\mathrm{Cr}(\mathrm{VI})$ are absorbed by CTS and CTS-DPPC nanospheres at $7 \mathrm{~h}$. The results display that the $\mathrm{Cr}(\mathrm{VI})$ adsorption capacity is improved with the addition of DPPC.

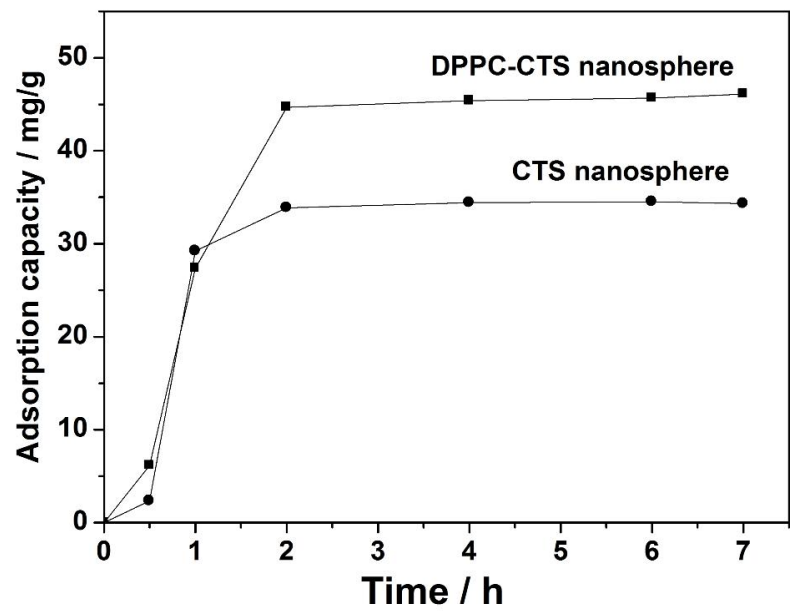

Figure 3 The effect of contact time on adsorption capacity of CTS and CTS-DPPC nanospheres.

Generally, the electrostatics is the popular adsorption mechanism between CTS and metal ions. ${ }^{[20]}$ In order to reveal the interaction between CTS-DPPC nanosphere and $\mathrm{Cr}(\mathrm{VI})$, the effect of $\mathrm{pH}$ and additional anions on the $\mathrm{Cr}(\mathrm{VI})$ adsorption performance by CTS and CTS-DPPC nanospheres was evaluated. As shown in Figure $4 a$, the $\mathrm{Cr}(\mathrm{VI})$ adsorption capacity increased at the range of $\mathrm{pH}=2-3$, and then decreased at the range of $\mathrm{pH}=3-6$ for both CTS and CTS-DPPC nanospheres at the acidic solution condition. The maximum $\mathrm{Cr}(\mathrm{VI})$ adsorption capacity at the $\mathrm{pH}=3$ is 33.8 and $44.6 \mathrm{mg} / \mathrm{g}$ for CTS and CTS-DPPC nanospheres, respectively. As we well known, in acid solution of $\mathrm{pH}=2-6$, the amino groups from CTS and DPPC molecules can be easily protonated to $\mathrm{NH}_{3}{ }^{+}$, but $\mathrm{Cr}(\mathrm{VI})$ always appears in the term of chromate, including $\left[\mathrm{HCrO}_{4}\right]^{-}$, $\left[\mathrm{CrO}_{2}\right]^{2-},\left[\mathrm{Cr}_{2} \mathrm{O}_{7}\right]^{2-},\left[\mathrm{Cr}_{3} \mathrm{O}_{10}\right]^{2-}$ and $\left[\mathrm{CrO}_{4}\right]^{2-}$ with the increasing $\mathrm{pH}$ from 2 to $6 .{ }^{[21]}$ Thus, a strong interaction could exist between the oxy anions of $\mathrm{Cr}(\mathrm{VI})$ and the positively charged surface of CTS and CTS-DPPC nanospheres. Moreover, $\mathrm{pH}$ is smaller or the acidity is higher, the protonation is stronger, which means that more $\mathrm{NH}_{3}{ }^{+}$could be existed at $\mathrm{pH} 2-3$. However, the $\left[\mathrm{HCrO}_{4}\right]^{-}$of chromate at $\mathrm{pH}=2$ could weak the electrostatic interaction between $\mathrm{Cr}(\mathrm{VI})$ and CTS or CTS-DPPC nanospheres because of weak ionic charge. With the further increasing $\mathrm{pH}$ from 3 to 6 , the ionic radius of chromate such as $\left[\mathrm{CrO}_{2}\right]^{2-},\left[\mathrm{Cr}_{2} \mathrm{O}_{7}\right]^{2-},\left[\mathrm{Cr}_{3} \mathrm{O}_{10}\right]^{2-}$ and $\left[\mathrm{CrO}_{4}\right]^{2-}$ could be enlarged, which may decrease the adsorption performance due to the steric hindrance. Therefore, these results may contribute to the maximum $\mathrm{Cr}(\mathrm{VI})$ adsorption capacity at the condition of $\mathrm{pH}=3$. Moreover, the more $\mathrm{NH}_{3}{ }^{+}$coming from both CTS and DPPC molecules leads to the stronger adsorption ability of CTS-DPPC nanospheres than that of CTS nanospheres. Furthermore, the effect of additional ions on the $\mathrm{Cr}(\mathrm{VI})$ adsorption performance by CTS and CTS-DPPC nanospheres was performed. The different anions $\left(\mathrm{Cl}^{-}, \mathrm{NO}_{3}{ }^{-}\right.$and $\mathrm{SO}_{4}{ }^{2-}$ and in the form of $\mathrm{NaCl}$, $\mathrm{NaNO}_{3}$ and $\mathrm{Na}_{2} \mathrm{SO}_{4}$ ) were added to the experimental system. As shown in Figure $4 \mathrm{~b}$, the inhibition of $\mathrm{Cr}(\mathrm{VI})$ adsorption by these anions are found with the order of $\mathrm{SO}_{4}{ }^{2-}>\mathrm{NO}_{3}{ }^{-}>\mathrm{Cl}^{-}$, in which the adsorption capacity are decreased by $9.84 \%, 4.56 \%$
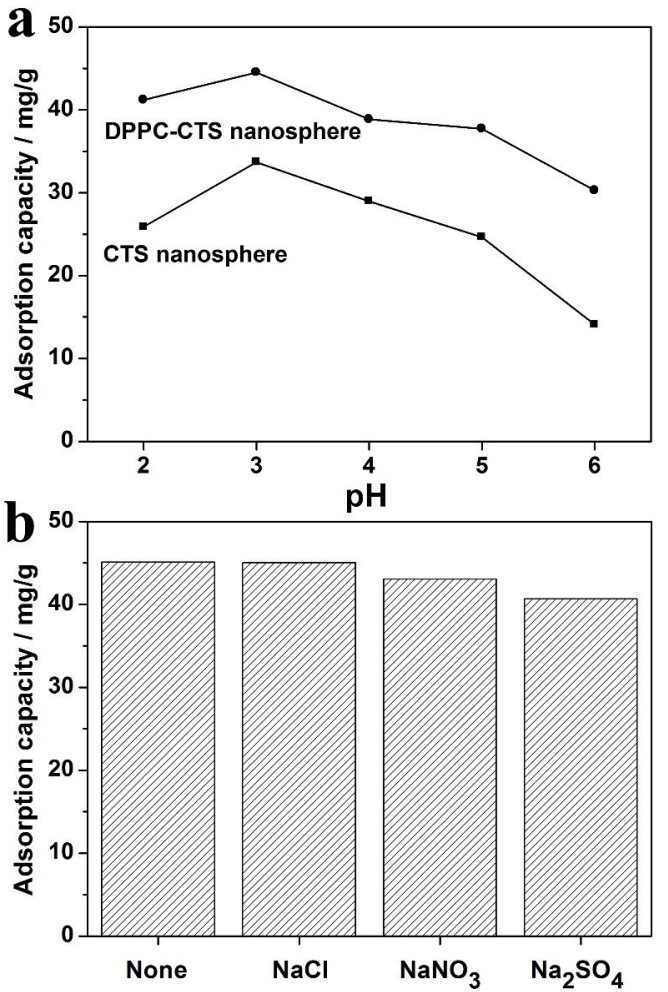

Figure 4 (a) The effect of pH on adsorption capacity of CTS and CTS-DPPC nanospheres and (b) the effect of additional anions on adsorption capacity of CTS-DPPC nanospheres. 
and $0.9 \%$. The results could contribute to the competing effect of the additional anions to interact with protonated amino groups through decreasing the number of the binding sites on CTS-DPPC nanospheres. Furthermore, the adsorption capacity is greatly affected by $\mathrm{SO}_{4}{ }^{2-}$ due to the crosslink of sulfate ions with protonated amino groups expect the strong competition between $\mathrm{SO}_{4}{ }^{2-}$ and $\mathrm{Cr}(\mathrm{VI}){ }^{[22]}$ In a word, the protonation of CTS and DPPC molecule, the charge and steric stabilization of chromate and the competing additional anion can influence the $\mathrm{Cr}(\mathrm{VI})$ adsorption performance.

Furthermore, the adsorption mechanism of CTS-DPPC nanospheres to $\mathrm{Cr}(\mathrm{VI})$ was proved by XPS. The XPS spectrum provided in Figure 5 a reveals carbon, oxygen, nitrogen, and chromium are the predominant elements observed on the surface from binding energies at $282 \mathrm{eV}$ (C 1s), $530 \mathrm{eV}$ (O 1s), 395 $\mathrm{eV}(\mathrm{N} \mathrm{1s})$, and550 eV (Cr 2p). Chromium is magnified in the spectra, which shows that $\mathrm{Cr} 2 \mathrm{p}_{3 / 2}$ peak at 576.64 and $2 \mathrm{p}_{1 / 2}$ peak at 586.24 are observed (Figure $5 b$ ). According to the previous report, the higher binding energy belongs to $\mathrm{Cr}(\mathrm{VI})$ and the lower binding energy peak to $\operatorname{Cr}(\mathrm{III}) .{ }^{[20]}$ Thus, the presence of two chromium peaks reveals that $\mathrm{Cr}(\mathrm{VI})$ is reduced partially. Therefore, there are two adsorption models including the physical adsorption and chemical adsorption, which comes from the electrostatic interaction between $\mathrm{Cr}(\mathrm{VI})$ and CTS or CTS-DPPC nanospheres and from the reduce reaction of $\mathrm{Cr}(\mathrm{VI})$ and functional groups in CTS and DPPC molecules. Furthermore, $\mathrm{N} 1 \mathrm{~s}$ and $\mathrm{O}$ 1s peaks of CTS-DPPC nanospheres before and after absorbing $\mathrm{Cr}(\mathrm{VI})$ are shown in Figures $5 \mathrm{c}$ and $5 \mathrm{~d} . \mathrm{N}$ $1 \mathrm{~s}$ peak at $340 \mathrm{eV}$ of CTS-DPPC nanospheres before absorbing $\mathrm{Cr}(\mathrm{VI})$ is split to two peaks at 399 and $402 \mathrm{eV}$. According to previous report, the two peaks assigned to $\mathrm{NH}_{2}$ or $\mathrm{NH}$ in the lower binding energy and $\mathrm{NH}_{3}{ }^{+}$in the higher binding energy, respectively. ${ }^{[23]}$ The significant change indicates the involvement of the amino group in the adsorption. Moreover, $O$ 1s peak at $532 \mathrm{eV}$ of CTS-DPPC nanospheres before absorbing $\mathrm{Cr}(\mathrm{VI})$ is shifted to $533 \mathrm{eV}$, which suggests that the hydroxyl groups are also involved in the adsorption. In other words, $\mathrm{Cr}$ ions of $\mathrm{Cr}(\mathrm{VI})$ and $\mathrm{Cr}(\mathrm{III})$ could be absorbed through their electrostatic interaction with $\mathrm{NH}_{3}{ }^{+}$and complexation with amino and hydroxyl groups.
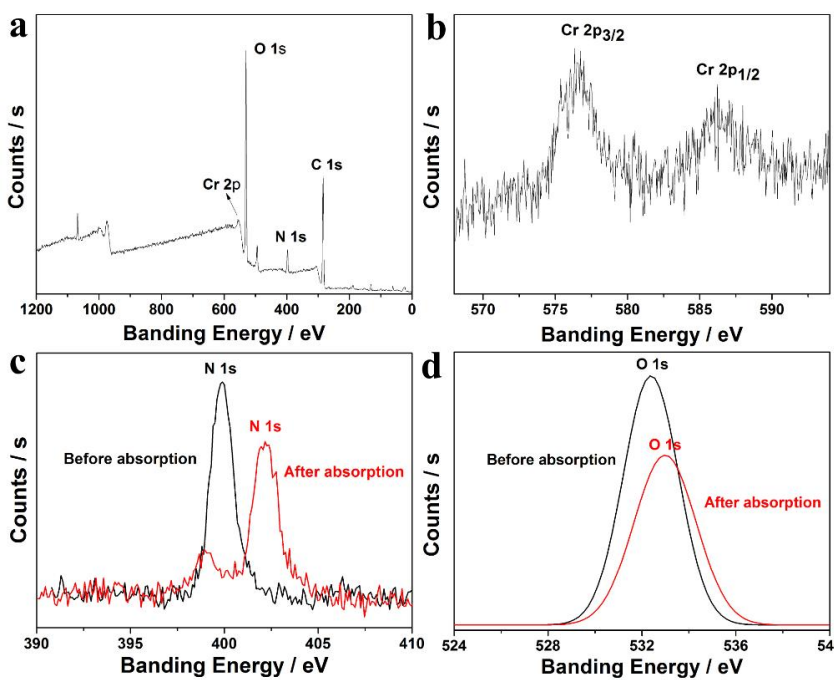

Figure 5 XPS spectra of (a) predominant elements and (b) $\mathrm{Cr}$ $2 p$ for DPPC after absorbing $\mathrm{Cr}(\mathrm{VI})$, (c) $\mathrm{N} 1 \mathrm{~s}$ and (d) $\mathrm{O}$ 1s for DPPC before and after absorbing $\mathrm{Cr}(\mathrm{VI})$.

As we well known, the increasing surface area is an effective route to improve the adsorption capacity. Thus, the electrospinning using the same DPPC-CTS content of
CTS-DPPC nanospheres in acetic acid as the spinning solution was performed. As shown in Figure 6a, the shape net-like composed by fibers with about $100 \pm 30 \mathrm{~nm}$ in diameter is obtained with the $20 \mathrm{kV}$ of spinning voltage and $8 \mathrm{~cm}$ of the collection distance. The product was used as a sorbent to remove $\mathrm{Cr}(\mathrm{VI})$. As shown in Figure $6 \mathrm{~b}$, the adsorption capacity of CTS-DPPC nanofibers to $\mathrm{Cr}(\mathrm{VI})$ is a continuous increase as a function of time at $0-7 \mathrm{~h}$. About $62.9 \mathrm{mg} / \mathrm{g}$ of $\mathrm{Cr}(\mathrm{VI})$ are $a b-$ sorbed after $7 \mathrm{~h}$ exposure, which is obvious higher than that of CTS-DPPC nanospheres. It is attributed to the increasing contact area, leading to the exposure of much more functional groups for acting with $\mathrm{Cr}(\mathrm{VI})$.
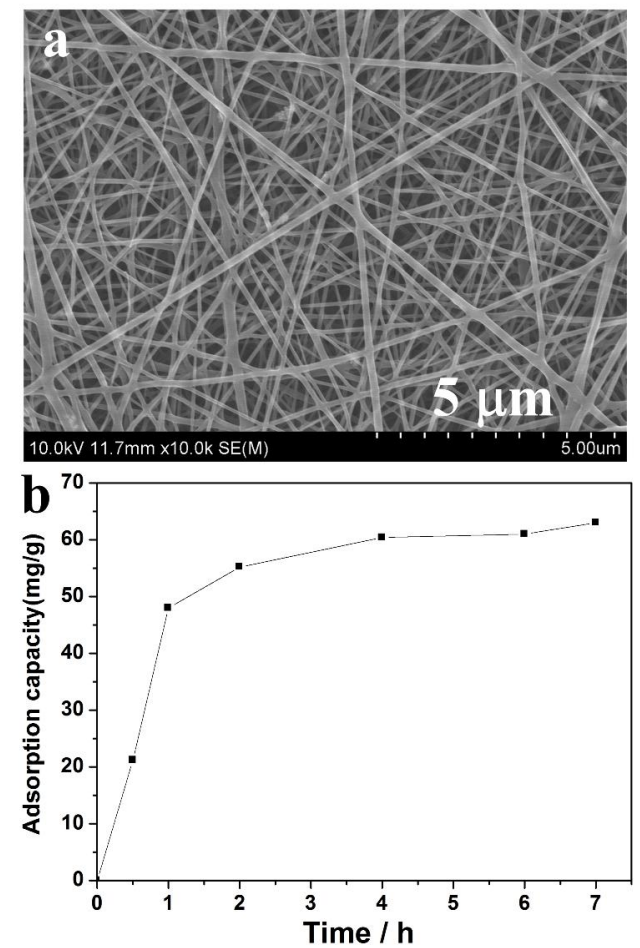

Figure 6 (a) SEM image and (b) the effect of contact time on adsorption capacity of electrospun DPPC-CTS nanofibers.

\section{Conclusions}

CTS-DPPC nanospheres and nanofibers obtained through CTS modified by DPPC in our experiment are used as a novel biosorbent for the application in $\mathrm{Cr}(\mathrm{VI})$ absorption. The results show that DPPC is helpful for CTS to remove metal ion, and 44.6 and $62.9 \mathrm{mg} / \mathrm{g}$ of $\mathrm{Cr}(\mathrm{VI})$ is absorbed by CTS-DPPC nanospheres and nanofibers after $2 \mathrm{~h}$ exposure, respectively. The effect of $\mathrm{pH}$ and additional anions on the $\mathrm{Cr}(\mathrm{VI})$ adsorption performance is evaluated, which reveals that the maximum adsorption capacity of $\mathrm{Cr}(\mathrm{VI})$ is at $\mathrm{pH}=3$ and anions affect $\mathrm{Cr}(\mathrm{VI})$ adsorption on CTS-DPPC nanofibers in the order of $\mathrm{SO}_{4}{ }^{2-}>\mathrm{NO}_{3}{ }^{-}>\mathrm{Cl}^{-}$. Furthermore, the adsorption mechanism of CTS-DPPC nanospheres to $\mathrm{Cr}(\mathrm{VI})$ was proved by XPS. The result shows that both amino and hydroxyl groups are involved in the adsorption of $\mathrm{Cr}(\mathrm{VI})$ ions on the CTS-DPPC nanospheres. All the results are indicative that CTS-DPPC nanospheres modified by DPPC can be used as a promising sorbents for $\mathrm{Cr}(\mathrm{VI})$ removal.

\section{Acknowledgement}

The work was supported by the Shanghai Science and Technology Development Fund (No. 17XD1421900). 


\section{References}

[1] Cogo, P. E.; Zimmermann, L. J.; Pesavento, R. Crit. Care Med. 2003, 31, 1532.

[2] Kendall, M. Physiol. Lung Cell Mol. Physiol. 2007, 293, 1053.

[3] Liacos, J. W.; Kam, W.; Delfino, R. J. Sci. Total Environ. 2012, 435.

[4] Jin, W.; Rong, L.; Liu, Y. Respirology 2013,18, 643.

[5] Postle, A. D.; Gonzales, L. W.; Bernhard, W. Lipid Res. 2006, 47, 1322.

[6] Dimos, V.; Haralambous, K. J.; Malamis, S. Environl. Sci. Technol. 2012, 42, 1977.

[7] Narayani, M.; Shetty, K. V. Environ. Sci. Technol. 2013, 43, 955

[8] Rakhunde, R.; Deshpande, L.; Juneja, H. D. Environ. Sci. Technol. 2012, 42, 776.

[9] Golder, A. K.; Chanda, A. K.; Samanta, A. N.; Ray, S. Sep. Purif. Technol. 2011, 76, 345.

[10] Jung, C.; Heo, J.; Han, J.; Her, N.; Lee, S. J.; Oh, J. Sep. Purif. Technol. 2013, 106, 63.

[11] Mohammadi, H.; Gholami, M.; Rahimi, M. Desalin. Water Treat. 2009, 9, 229.

[12] Sag, Y.; Aktay, Y. Biochem. Eng. J. 2002, 12, 143.

[13] Ahmad, A. L.; Sumathi, S.; Hameed, B. H. Sci. Technol. 2004, 22, 75.
[14] Hassan, M. L.; Fadel, S. M.; El-Wakil, N. A. J. Appl. Polym. Sci. 2012, 125, 216.

[15] Boddu, V. M.; Abburi, K.; Talbott, J. L.; Smith, E. D. Environ. Sci. Technol. 2006, 97, 2377.

[16] Calvo, P.; Remunan-Lopez, C.; Vila-Jato, J. L. Pharm. Res. 1997, $14,1431$.

[17] Masri, M. S.; Reuter, F. W.; Friedman, M. J. Appl. Polym. Sci. 1974, $18,675$.

[18] Liu, H.; Zhang, Y. L.; Han, Y. Z. Colloid Surf. B 2015, 131, 12.

[19] Wang, H. C. Shanghai Science and Technology Press, Shanghai, China, 1987, p. 8.

[20] Miretzky, P.; Cirelli, A. F. J. Hazard. Mater. 2009, 167, 10.

[21] Udaybhaskar, P.; lyengar, L.; Rao, A. V. S. P. J. Appl. Polym. Sci. 1990, 39, 739

[22] Anjali, D. D.; Smitha, B.; Sridhar, S.; Aminabhavi, T. J. Membr. Sci. 2006, 280, 45.

[23] Boddu, V. M.; Abburi, K.; Talbott, J. L.; Smith, E. D. Enviro. Sci. Technol. 2003, 37, 4449 .

Received January 13, 2019

Accepted February 22, 2019 